\title{
Analysis of the Electromagnetic Environment in the Aircraft with Airborne VLF DTWA
}

\author{
Su Liyuan*, Ma Yao, Guo Fei, Duan Yantao, Gao Cheng \\ National Key Laboratory on Electromagnetic Environmental Effects and Electro-Optical Engineering \\ Army Engineering University of PLA \\ Nanjing, China \\ song_li@126.com* corresponding author; mayao_84@aliyun.com; \\ ariessa@126.com; dcmchdyt@126.com; gc6868@163.com
}

\begin{abstract}
The airborne VLF (Very Low Frequency) transmitter has high power and wide communication range. However, due to the complex antenna configuration and the fuselage as a part of the antenna, the internal electromagnetic environment of the aircraft is very harsh for airborne equipment and personnel. In this paper, the electromagnetic environment generated by airborne DTWA (Dual Trailing Wire Antenna) is simulated. The protective effect of simple protective means is simulated and analyzed, and the influence of airborne electromagnetic field on airborne personnel and equipment is evaluated. The conclusions are helpful for the further research on the application of airborne VLF communication system.
\end{abstract}

Keywords: VLF, DTWA, Electromagnetic Environment, Simulation, Aircraft.

\section{Introduction}

Airborne very low frequency (VLF) radio has become a major survivable submarine communication system [1]. It has great transmitted power and the ability for long distance communication. At the same time, it can meet the requirements of rapid deployment and security hiding. DTWA (Dual Trailing Wire Antenna) is the main part of the airborne VLF radio station, which consists of two wire antennas: a short antenna connected with the transmitter which is integrated in the cabin, and a long antenna connected with the airframe [2]. The transmitter's working frequency is commonly between $10 \mathrm{kHz}$ to $30 \mathrm{kHz}$ [3]. The airplane should fly in circles to keep the long antenna in a larger vertical degree [4]. Due to the flight attitude, the form of the antenna is irregular, which has both horizontal and vertical components and the radiation field in the space is very complicated [5].

In previous work, we established the VLF transmitting system model and the carrier model based on the IL-76 transport aircraft. The skin of the aircraft was mainly composed of metal and a spot of composite materials [6], as shown in Fig.1, which included many precise structures, such as a cockpit windshield and the gap of the cabin door, etc. Considering the circling motion of the aircraft, we build the antenna model as Fig.2. The transmitter operating frequency was set as $30 \mathrm{kHz}$, and the power was $200 \mathrm{~kW}$. Basing on this model, we calculated the electromagnetic field distribution and the induced current of the cables inside the aircraft cabin. The conclusions indicated that when there was no protection, the inducted electromagnetic fields generated by the transmitter and trailing antenna combined in the aircraft structure were so strong that the airborne equipment could be interfered or even damaged.

In this paper, we do a further study on the electromagnetic environment in the aircraft with airborne VLF transmitting system, the protection against windshield of the cockpit and the shielding method in the main cabin are analyzed based on the numerical simulation software FEKO. 


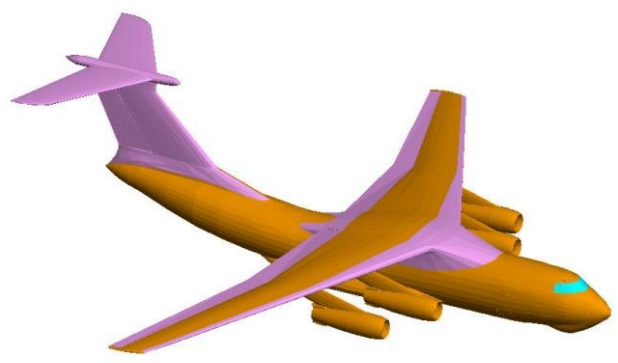

Fig. 1: The carrier aircraft model.

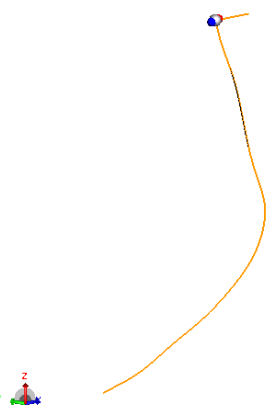

Fig. 2: The DTWA model.

\section{The Field Distribution in the Cockpit}

A large number of avionics is installed in the cockpit, so it is necessary to ensure that the electric field of the cockpit meets the sensitivity requirements. As shown in Fig.3, the windshield almost have no shielding effect if it is made of common glass, The strength of the electric field in the front of the cockpit is higher than $200 \mathrm{~V} / \mathrm{m}$, and even reach $1000 \mathrm{~V} / \mathrm{m}$. The magnetic field in the cockpit is higher than $0.1 \mathrm{~A} / \mathrm{m}$, and it can be over $2.5 \mathrm{~A} / \mathrm{m}$ near the edge of the windshield, so the instruments installed in the front of cockpit might be interfered or damaged by the intense electromagnetic field.

There must be a proper protection method against such strong field. A common method is coating the cockpit glass with a conductive layer. A silver ion conductive coating of 20 microns thick on the windscreen is set, and the electromagnetic field distribution in the cockpit is shown in Fig.4.

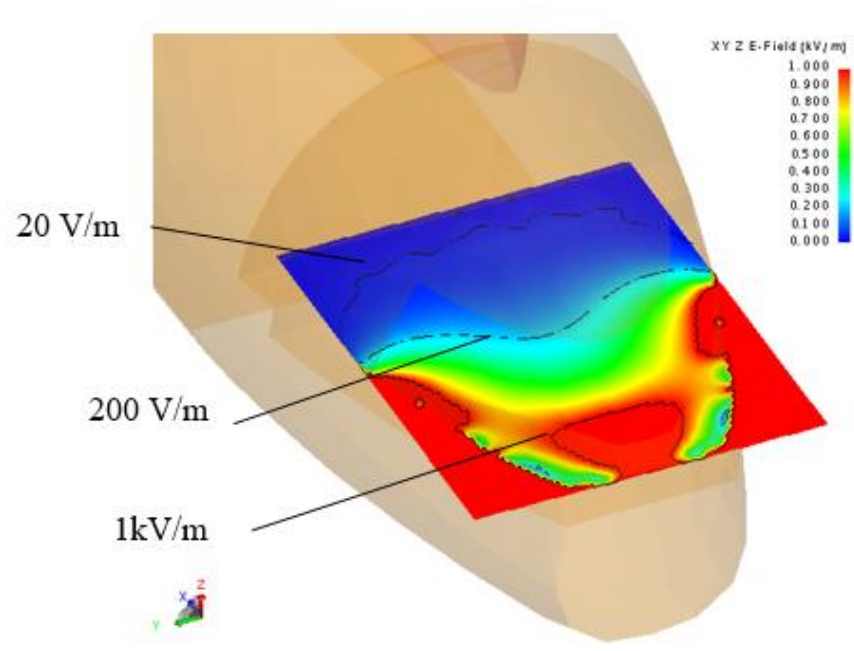

(a) the electric field

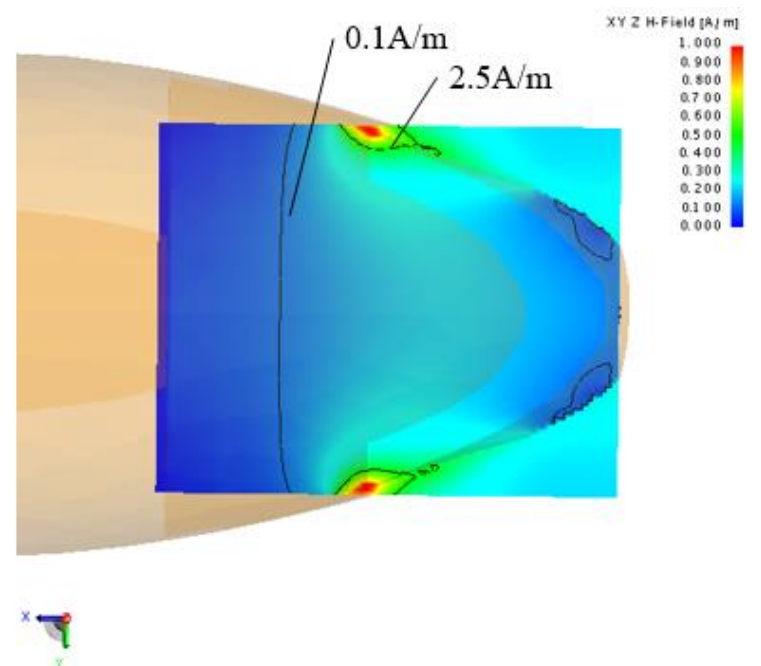

(b) the magnetic field

Fig. 3: The field distribution of the cockpit. 


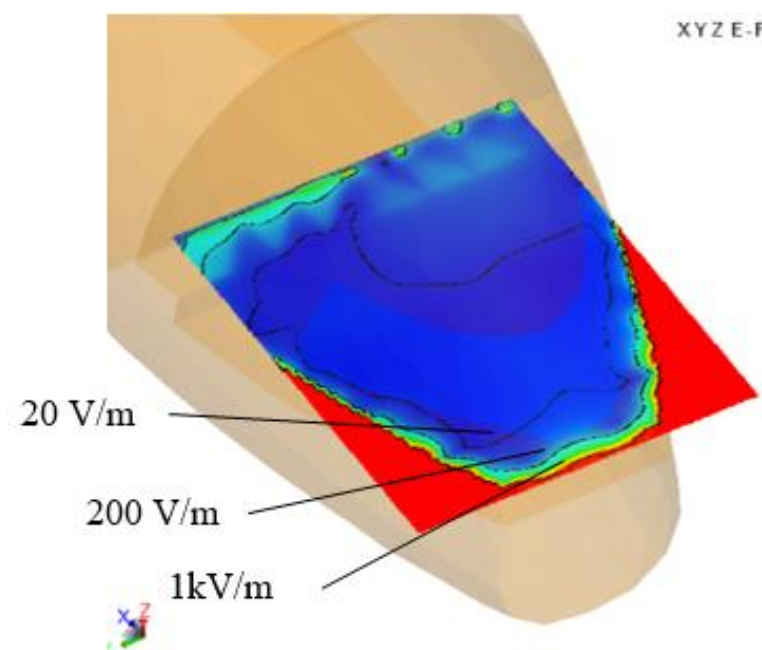

(a) the electric field

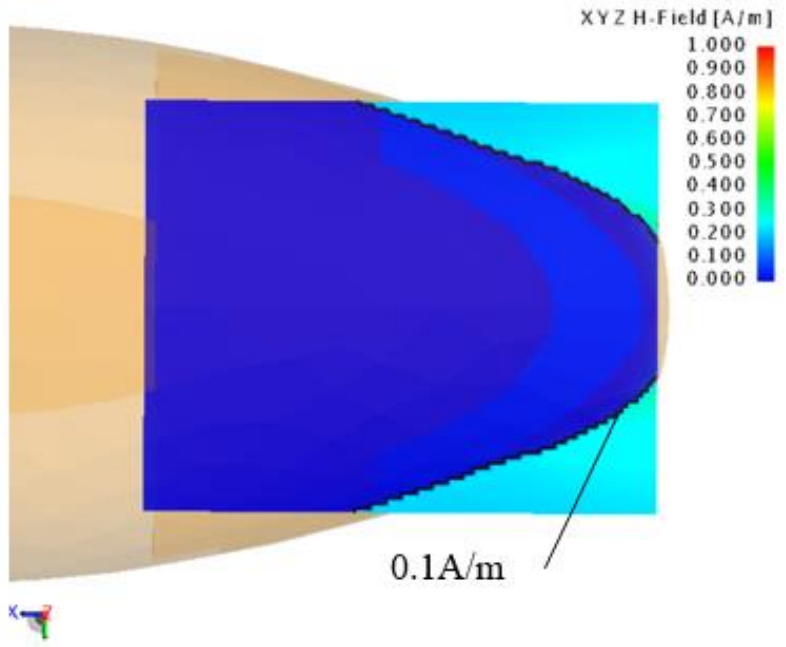

(b) the magnetic field

Fig. 4: The field distribution of the cockpit (when the windshield has been overlay with a conductive coating).

As we can see, the electric field strength in the cabin is greatly reduced after the protection, and it is less than $20 \mathrm{~V} / \mathrm{m}$ in $\mathrm{most}$ areas. Because the conduction continuity of the skin is maintained by the coating layer, the magnetic field strength is weakened to a very low level. Thus the shielding effectiveness is greatly optimized.

\section{The Field Distribution in the Main Cabin}

In the VLF carrier, the equipment module is usually arranged in the front part of the main cabin, and the important airborne electronic equipments and systems are installed in this area, which is close to the installation position of the VLF transmitter. When there are no targeted protection measures, the field strength is far beyond the general sensitivity of the airborne equipments. Shielding is the most effective protection method to be considered. It's simple and practicable to separate the equipment compartment from the main cabin by a metal partition. Fig.5 and Fig.6 show the field distribution before and after the metal partition installed on the floor.

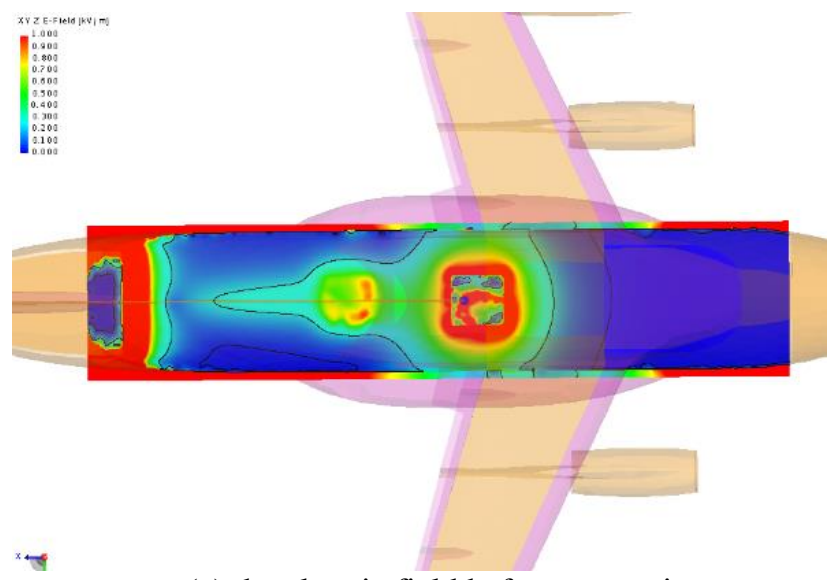

(a) the electric field before separating

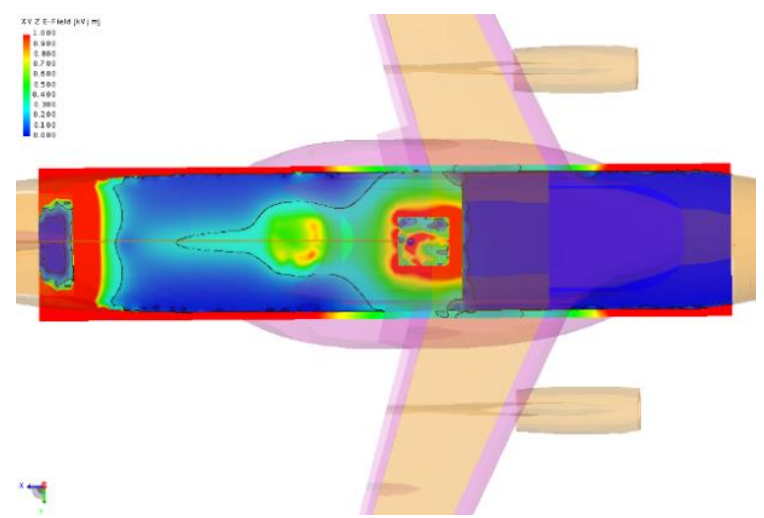

(b) the electric field after separating 


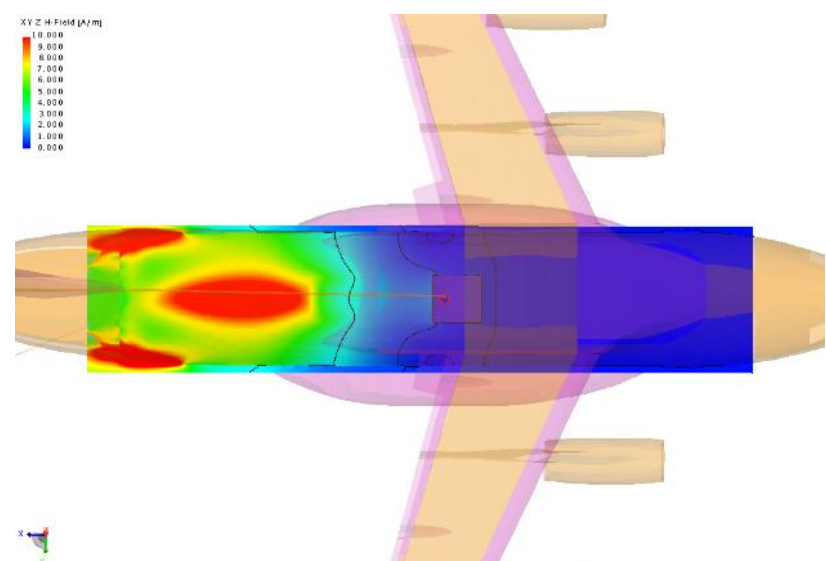

(c) the magnetic field before separating

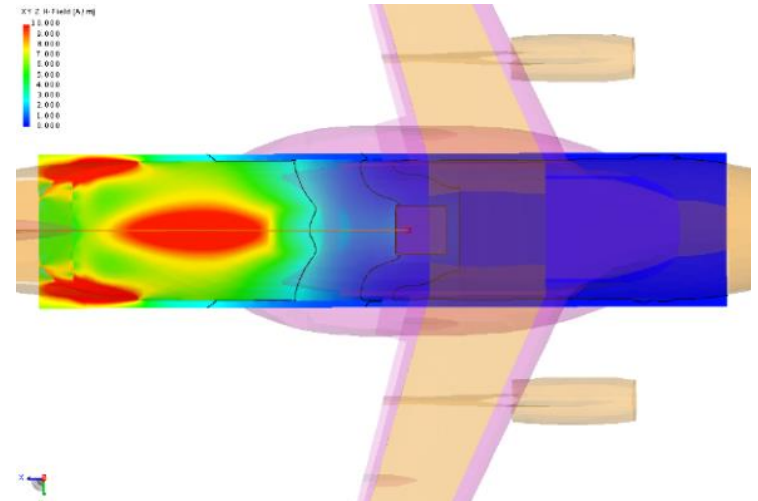

(d) the magnetic field after separating

Fig. 5: The field distribution in the main cabin (before and after the metal partition is installed).

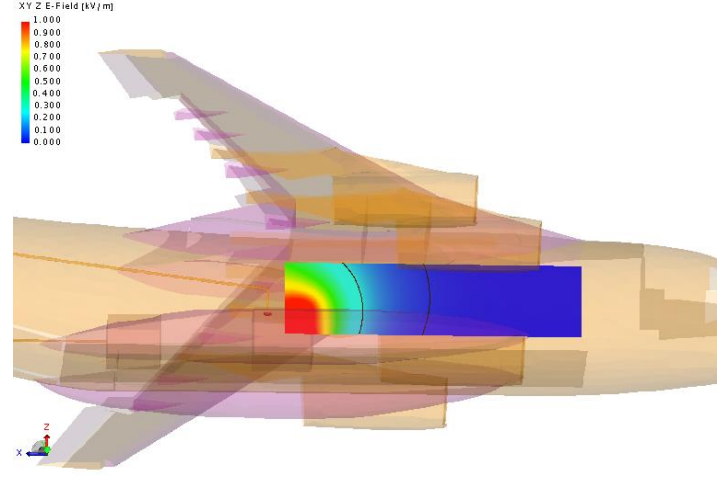

(a) before separating

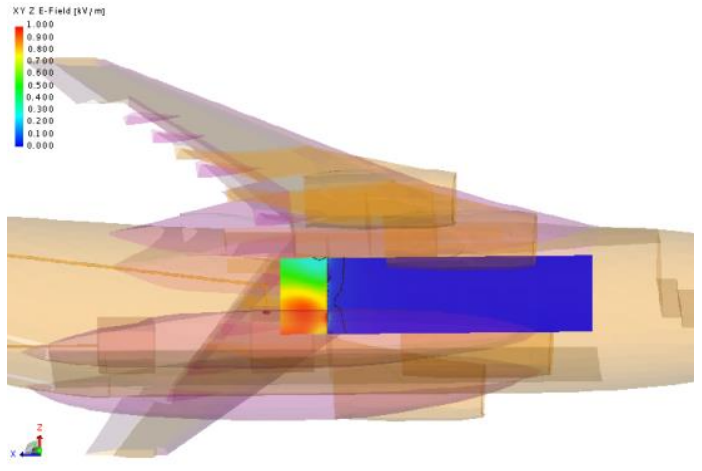

(b) after separating

Fig. 6: The electric field distribution in the area of the equipment installed (side view).

The calculating results in Fig.7 shows that the metal partition can also have a good shielding effect even if it does not have a fully enclosed compartment with the fuselage (there is no isolation below the floor). The electric field intensity in the partition is reduced from hundreds of volts to dozens of volts per meter, and the magnetic field intensity in the partition is less than $0.1 \mathrm{~A} / \mathrm{m}$.

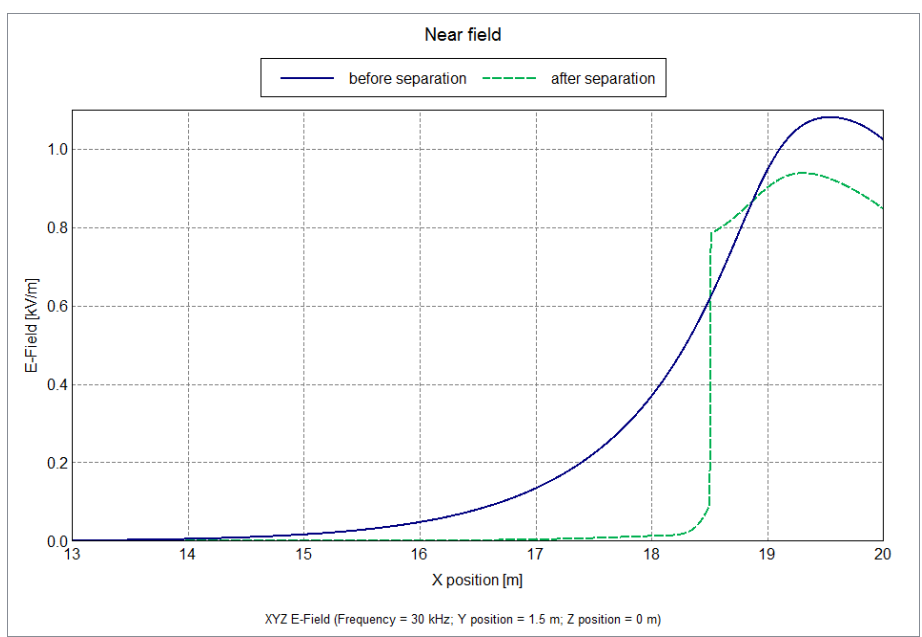

Fig. 7: Comparison of the electric field intensity along the central axis of the main cabin. 


\section{Analysis of the Impedance Characteristics of the Electromagnetic Field in the Carrier}

The fundamental of the electromagnetic environment in the aircraft is $30 \mathrm{kHz}$. Because the carrier is part of the whole antenna system, the cabins are in the near field. So the impedance characteristics of the field should be considered when designing shielding. The wave impedance of free space can be calculated by the equation $Z=E / H$. So the wave impedance can be estimated according to the calculation results. The electric field intensity and magnetic field intensity of the typical positions in the cockpit and the main cabin are selected to estimate the wave impedance, shown in Table 1.

Table 1: The electromagnetic field strength and wave impedance of the typical positions in the aircraft.

\begin{tabular}{|c|c|c|c|}
\hline $\begin{array}{c}\text { Position (the } \\
\text { coordinates of } \\
\text { the points) }\end{array}$ & $\begin{array}{c}\text { Electric field } \\
\text { strength } \\
(\mathrm{V} / \mathrm{m})\end{array}$ & $\begin{array}{c}\text { Magnetic field } \\
\text { strength } \\
(\mathrm{A} / \mathrm{m})\end{array}$ & $\begin{array}{c}\text { Wave } \\
\text { impedance }(\Omega)\end{array}$ \\
\hline$(4,0,0.5)$ & 1150 & 0.166 & 6903 \\
\hline$(5,0,0.5)$ & 382 & 0.183 & 2081 \\
\hline$(6,0,0.5)$ & 142 & 0.140 & 1011 \\
\hline$(9,0,0)$ & 676 & 0.007 & 96850 \\
\hline$(12,0,0)$ & 790 & 0.074 & 10748 \\
\hline$(14,0,0)$ & 777 & 0.258 & 301 \\
\hline$(16,0,0)$ & 762 & 0.916 & 83 \\
\hline$(22,0,0)$ & 1143 & 16.7 & 68 \\
\hline$(28,0,0)$ & 834 & 17.5 & 48 \\
\hline$(32,0,0)$ & 590 & 12.3 & 48 \\
\hline
\end{tabular}

From Table 1 we can see that the electromagnetic field belongs to low frequency high impedance field in most area of the cockpit and the front area of the main cabin; and the field near the antenna from the transmitter to the tail belongs to the low frequency low impedance field. The absorption loss of low frequency field is very small, and the reflection loss of low impedance field is also small, so the shielding against the low frequency low impedance field is difficult. When designing shield the wave impedance should be taken into account according to the installation position.

\section{Conclusion}

In this paper, the electromagnetic fields in cabins of the transport aircraft with airborne VLF transmitting system are analyzed. From the simulation results, we can evaluate the impact of airborne VLF communication systems on equipment and personnel.

\subsection{The Impact on the Equipments in the Aircraft}

When there is no protection, the electric field intensity in most area of the cabins is higher than $200 \mathrm{~V} / \mathrm{m}$, which is far beyond the radiation sensitivity limit of airborne electronic equipments [7]. Through the protection methods such as coating windshield with conductive layer and setting metal partition, the inside electric field environment can be improved to a certain extent, but specific protective measures are still needed for the airborne equipments.

The magnetic field intensity is generally hundreds of milliamperes per meter, which is basically safe for the airborne electronic equipments. But in the area near the seam of fuselage skin, bent corners and so on, where the induced current distributed relatively concentrated, the magnetic field strength will increase sharply. So we should pay attention to avoiding these areas when installing the airborne equipments.

The electromagnetic fields in cabins are very complex. The impedance characteristic is varying with the relative distance from the transmitter and the antenna wire. It is high impedance field in most area of the cabins. But near the antenna wire, it is low impedance field. 


\subsection{The Impact on the Personnel in the Aircraft}

According to the standards of IEEEC95.1-2005 [8] and ICNIRP-2010 [9], the electric and magnetic field strength limits of occupational exposure and public exposure are listed in Table 2.

Table 2: The limits of occupational exposure and public exposure.

\begin{tabular}{|c|c|c|c|c|c|}
\hline & \multirow{2}{*}{$\begin{array}{c}\text { Frequency } \\
\text { range } \\
\end{array}$} & \multicolumn{2}{|c|}{ IEEEC95.1-2005 } & \multicolumn{2}{c|}{ ICNIRP-2010 } \\
\cline { 3 - 6 } & $\begin{array}{c}\text { Electric field } \\
(\mathrm{V} / \mathrm{m})\end{array}$ & $\begin{array}{c}\text { Magnetic } \\
\text { field (A/m) }\end{array}$ & $\begin{array}{c}\text { Electric field } \\
(\mathrm{V} / \mathrm{m})\end{array}$ & $\begin{array}{c}\text { Magnetic } \\
\text { field (A/m) }\end{array}$ \\
\hline $\begin{array}{c}\text { Occupational } \\
\text { exposure }\end{array}$ & $3 \sim 100$ & 1842 & 490 & 170 & 80 \\
\hline $\begin{array}{c}\text { public } \\
\text { exposure }\end{array}$ & $3 \sim 100$ & 614 & 163 & 83 & 21 \\
\hline
\end{tabular}

For the airborne personnel, the electric field strength in the cabins is beyond the public exposure limits and even the occupational exposure limits in some area, so if there is no reliable protection, the electromagnetic environment is unsafe for the airborne personnel.

The electromagnetic environment in the aircraft arise from the near field of the airborne VLF transmitting system is very harsh, the specific protection technology still need further research.

\section{References}

[1] J. Hu, Y. Cai, "Very Low Frequency Airborne Antenna," Modern Communication Technology, vol. 2, pp. 37-45, 1999.

[2] D. Wu, "Analysis of dual trailing wire antenna's wire model," Ship Electronic Engineering, vol. 29, pp. 68-71, 2009.

[3] J. Chen, "Simulation analysis of Airborne VLF wire antennas based on FEM," Radio Wave Guard. pp.71-74, 2013.

[4] W. Fan, S. Zhang, X. Han, "Radiate Electromagnetic Field for Airborne VLF Trailing Antenna Based on Theory of Wave Guide," Chinese Journal of Radio Science., vol. 1, pp. 114-120, 2015.

[5] L. Chen, L. Yanyan, "Electromagnetic Compatibility Design and Engineering Implementation of Transmitter with High Motor Power and Ultra-Low Frequency," Journal of Henan Science and Technology. vol. 1, pp. 20-23, 2015.

[6] A. A. Baker, S. E. Dutton, D. W. Kelly, Composite materials for aircraft structures, 2nd ed. American Institute of Aeronautics and Astronautics, vol. 43, pp. 213-223, 2004.

[7] MIL-STD-461F Requirements for the Control of Electromagnetic Interference Characteristics of Subsystems and Equipment, 2007.

[8] IEEEC95.1-2005 IEEE Standard for Safety levels with ResPectto Human Exposure to Radio Frequency Electromagnetic Fields 3kHz to 300GHz, 2005.

[9] ICNIRP-2010 "ICNIRP Guidelines for Limiting exposure to Time-Varying Electric and Magnetic Fields (1Hz100kHz)," Health Physics, vol. 99, no. 6, pp. 818-836, 2010. 